\title{
Design Considerations for Battery-Powered Electronics ${ }^{*}$
}

\author{
Massoud Pedram Qing Wu \\ Department of Electrical Engineering-Systems \\ University of Southern California \\ Los Angeles, CA 90089 \\ \{pedram,qwu\}@usc.edu
}

\begin{abstract}
In this paper, we consider the problem of maximizing the battery life (or duration of service) in battery-powered CMOS circuits. We first show that the battery efficiency (or utilization factor) decreases as the average discharge current from the battery increases. The implication is that the battery life is a super-linear function of the average discharge current. Next we show that even when the average discharge current remains the same, different discharge current profiles (distributions) may result in very different battery lifetimes. In particular, the maximum battery life is achieved when the variance of the discharge current distribution is minimized. Analytical derivations and experimental results underline importance of the correct modeling of the battery-hardware system as a whole and provide a more accurate basis (i.e., the battery discharge times delay product) for comparing various low power optimization methodologies and techniques targeted toward battery-powered electronics. Finally, we calculate the optimal value of $V_{d d}$ for a battery-powered VLSI circuit so as to minimize the product of the battery discharge times the circuit delay.
\end{abstract}

\section{INTRODUCTION}

With the rapid progress in the semiconductor technology, the chip density and operation frequency have greatly increased, making the power consumption in digital circuits a major concern for VLSI designers. High power consumption reduces the battery life in portable devices. Therefore, the goal of low-power design for battery-powered devices is to extend the battery lifetime while meeting the performance requirement.

An effective method for low-power design is to reduce the supply voltage while satisfying the performance constraints by a combination of architectural and circuit optimization techniques. For a fixed supply voltage level, low power techniques target at reducing the average current drawn by the circuit [7]. Voltage scaling techniques, on the other hand, scale the supply voltage to reduce power dissipation. These techniques can be divided into static voltage scaling [1][2] and dynamic voltage scaling [3]. The effectiveness of these techniques can be evaluated by using appropriate metrics, i.e., power, energy, delay, or energy-delay product. These metrics can be used in different applications (depending on the design requirements) to guide optimization toward the best solution. It has been argued in [2] that the energydelay product is more relevant for comparing various low power design methodologies and techniques.

\footnotetext{
* This work is supported in part by an NSF PFF/PECASE award under contract No. MIP-9628999.
}

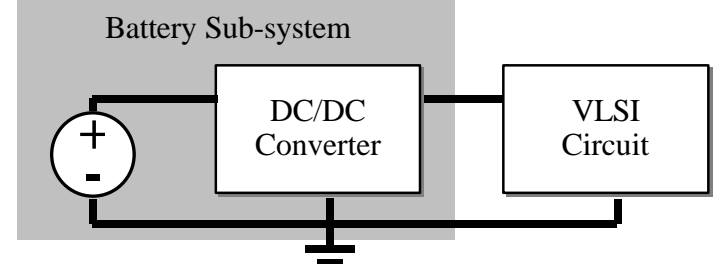

Figure 1 An integrated model of battery-powered system

As shown in Figure 1, a battery-powered digital system (which is typically present in portable electronic devices consists of the VLSI circuit, the battery cell, and the DC/DC converter. In spite of the fact that the goal of low-power design for portable electronics is to extend the battery service time, discussions of low-power design metrics and methodologies have entirely focused on the VLSI circuit itself, assuming that the battery sub-system is an ideal source that outputs a constant voltage and stores/delivers a fixed amount of energy [4]. In reality, the energy stored in a battery may not be extracted/used to the full extent. In some situations, even 50\% energy delivery is not possible. This phenomenon is caused by the fact that the "actual capacity" of the battery depends strongly on the mean value and the profile (distribution) of the current discharged from the battery. More precisely, higher portion of the battery capacity is wasted at higher discharge current. High rate (current) discharge can indeed cause dramatic waste of the initial capacity (energy storage) of the battery. Furthermore, even for the same mean value of discharge current, the battery efficiency may change by as much as $25 \%$ as a result of the discharge current profile.

In this paper, we will show that for a given battery, the amount of energy that can be used by the VLSI circuit is a function of the current discharge rate of the VLSI circuit ${ }^{1}$. In particular, the battery life does not have a simple linear relationship with the power consumption of the circuit. For example, a $2 \mathrm{X}$ increase in circuit power consumption may cause a $3 \mathrm{X}$ reduction in the battery lifetime, compared with the $2 \mathrm{X}$ reduction in the ideal case [6]. Therefore, for portable battery-powered electronics, the appropriate metric to guide various design optimizations is the battery discharge - delay product, and not simply the energy-delay product [2]. We will also show that, because of the dependence of the battery capacity on the discharge current, current discharge with same average value, but different profiles (distributions) may lead to different battery lifetimes.

This paper is organized as follows. Section II provides some background on battery design and engineering. Section III gives an analysis of the relationship between the current profile and the battery life. Section IV considers the problem of optimal supply voltage selection. Sections V and VI present experimental results and conclusions.

\footnotetext{
${ }^{1}$ Some energy is also wasted in the DC/DC converter. This is relatively small and independent of the output current demand for a well-designed DC/DC converter [5].
} 


\section{BACKGROUND}

\section{A. Battery Overview}

Many different types of batteries are used in a wide range of applications [5]-[16]. They can be divided into the primary batteries (non-rechargeable) and the secondary batteries (rechargeable). Batteries can also be classified based on the electrochemical material used for their electrodes or the type of their electrolytes, e.g., Lead-acid, Ni-Cd, Ni-Zn, Ag-Zn, Zn-Air, Nickel-Metal Hydride, Lithium-Ion, Lithium-Polymer, etc. Among these, the Nickel-Metal Hydride battery and the LithiumIon battery are currently the most popular secondary batteries for portable electronic devices, ranging from cellular phones to notebook computers.

Figure 2 shows the internal structure of a typical rechargeable lithium battery. The battery consists of the lithium foil anode, the composite cathode, and the electrolyte that serves as an ionic path between electrodes and separates the two materials. During discharge, the electrochemical process involves the dissolution of lithium ions at the anode, their migration across the electrolyte and their insertion within the crystal structure of the cathode. Positive current flows in the opposite direction in the external circuitry. Applying electrical recharging can reverse the reaction; hence the battery can be used for multiple times (normally several hundred times).

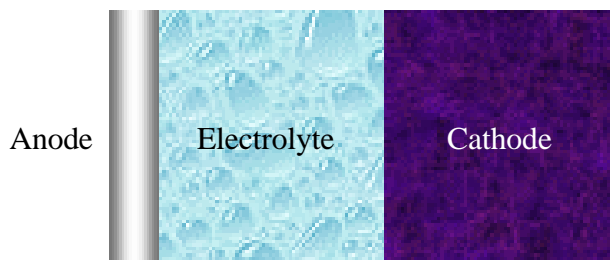

Figure 2 The internal structure of a Lithium battery.

\section{B. DC/DC Converters}

Figure 3 (taken from [5]) shows the block diagram of the Buck Converter of a high-efficiency DC/DC converter that can be integrated on the chip. The control circuit of the DC/DC converter is not shown due to space limitation. Node $V_{0}$ is the input of the $\mathrm{DC} / \mathrm{DC}$ converter that is connected to the positive electrode of the battery. Node $V_{d d}$ is the output of the DC/DC converter and is connected to the VLSI circuit. The control circuit is used to adaptively generate the switching signals for the Buck Converter such that the voltage at $\mathrm{V}_{d d}$ is stabilized at the target supply voltage for the VLSI circuit.

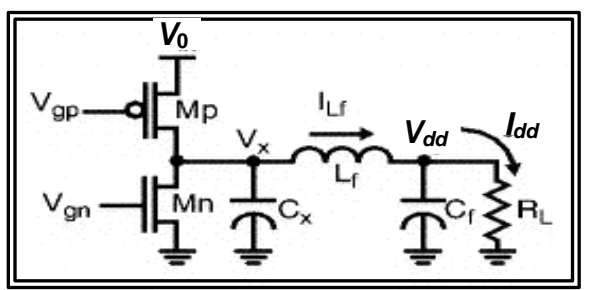

Figure 3 The Buck Converter of a DC/DC converter.

If we define $\eta$ as the conversion efficiency of the DC/DC converter, we have:

$$
\eta \cdot V_{0} \cdot I_{0}=V_{d d} \cdot I_{d d}
$$

where $I_{0}$ and $I_{d d}$ are average input and output current of the DC/DC converter over some period of time. $V_{0}$ and $V_{d d}$ are similarly defined. Notice that $V_{0}$ and $I_{0}$ are also the output voltage and current of the battery, $V_{d d}$ and $I_{d d}$ are also the supply voltage and current for the VLSI circuit.

\section{Battery Capacity and Efficiency}

One important characteristic of the battery is that some amount of energy will be wasted when the battery is delivering the energy required by the circuit [8]-[17]. In analytical form, given a fixed battery output voltage, if the circuit current requirement for the battery is $I$, the actual current which is taken out of the battery is:

$$
I^{a c t}=\frac{I}{\mu}, \quad 0 \leq \mu \leq 1
$$

where $\mu$ is called the battery efficiency (or utilization) factor. $I^{a c t}$ is always larger than or equal to $I$.

Defining $C A P_{0}$ as the amount of energy that is stored in a new (or fully charged) battery and $C A P^{a c t}$ as the actual energy that can be used by the circuit, Eqn. (2.2) is equivalent to:

$$
C A P^{a c t}=C A P_{0} \cdot \mu, \quad 0 \leq \mu \leq 1
$$

The efficiency factor $\mu$ is a function of discharge current $I$ :

$$
\mu=f(I)
$$

where $f$ is a monotonic-decreasing function [5]. Only the lowfrequency part of the current is relevant to changing the battery efficiency [16]. Therefore, $I$ must be the average output current of the battery over some period of time. This time is represented as $N \cdot T$, where $N$ is some positive integer and $T$ is the clock cycle. $N \cdot T$ may be as large as a few seconds [16]. The actual capacity of the battery decreases when the discharge current increases.

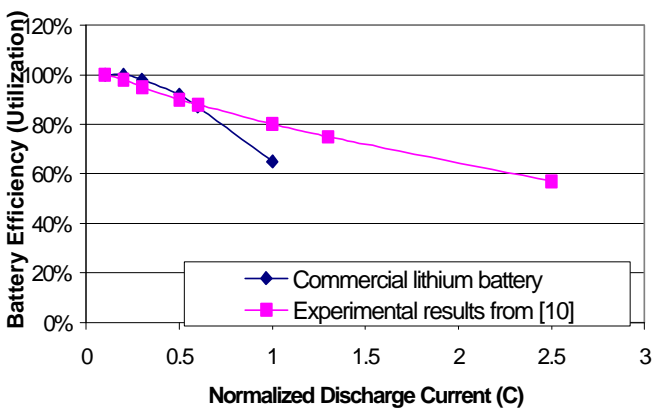

Figure 4 Discharge capacity of a commercial lithium battery.

Figure 4 shows the efficiency factor versus discharge current curves extracted from the data sheet of a commercial Lithium battery [17] and the experimental results from [12]. Similar curves exist for other lithium batteries [8][9][15] and for NiMH batteries [14][15].

To obtain an analytical form for the discussions in the remainder of the paper, two simple functions are used to approximate the battery efficiency factor:

or

$$
\begin{aligned}
& \mu=1-\beta \cdot I \\
& \mu=1-\gamma \cdot I^{2}
\end{aligned}
$$


where $\beta$ and $\gamma$ area positive constant numbers.

In our experience, either Eqn. (2.5) or Eqn. (2.6) provide good modeling for the capacity-current relation of Lithium batteries as long as the appropriate value of $\beta$ or $\gamma$ is chosen.

\section{Notation}

Before we present our analytical results in Section III, we give some useful notation:

$T$ : Clock cycle time for one operation

$V_{0}$ : Output voltage of the battery

$I_{0}$ : Average output current of the battery over time $N \cdot T$

$V_{d d}$ : Supply voltage of the circuit

$I_{d d}$ : Average supply current of the circuit over time $N \cdot T$

$\mu$ : Efficiency factor of the battery

$\eta$ : Efficiency of the DC/DC converter

$P^{i d e}$ : Ideal battery power required by the circuit

$P^{a c t}$ : Actual battery power needed for output power of $P$

$E^{\text {ide }}$ : Ideal energy needed to complete an operation

$E^{a c t}$ : Actual battery energy needed to complete an operation

$C A P_{0}$ : Total energy stored in a new battery

DOS: Duration of service, or battery lifetime, equals to $C P A_{0}$ divided by $P^{a c t}$

\section{$B D$ : Battery discharge}

Notice that $V_{0}, V_{d d}$ and $\eta$ are nearly constant during the circuit operation.

\section{CURRENT PROFILE VERSUS DURATION OF SERVICE}

We will show that, even with the same power consumption, the battery lifetime is different for different circuit current profile (also referred to as the current distribution).

Assume that, during the circuit operation, the magnitude of the average circuit current ${ }^{2} I_{d d}$ follows a certain probability density function $p_{1}\left(I_{d d}\right)$, and the battery current $I_{0}$ follows the density function $p_{2}\left(I_{0}\right)$. From Eqn. (2.1) we know that $p_{1}$ and $p_{2}$ have a linear relationship, the only difference is the scales of the axes. Therefore, we will focus on the relation between $p_{2}$ and the battery life; our derivations are equally applicable to $p_{1}$.

\section{A. Actual power and duration of service}

Let $I_{0}^{\text {ave }}$ be the mean value of distribution $p_{2}\left(I_{0}\right)$, we can write the ideal power consumption of the circuit as:

$$
\begin{aligned}
P^{i d e} & =\int_{I_{0, M I N}}^{I_{0, M A X}} V_{0} \cdot I_{0} \cdot p_{2}\left(I_{0}\right) d I_{0} \\
& =V_{0} \cdot \int_{I_{0, M I N}}^{I_{0, M A X}} I_{0} \cdot p_{2}\left(I_{0}\right) d I_{0}=V_{0} \cdot I_{0}^{\text {ave }}
\end{aligned}
$$

The actual power consumption of the circuit can be written as:

$$
P^{a c t}=V_{0} \cdot \int_{I_{0, M I N}}^{I_{0, M A X}} \frac{I_{0}}{\mu\left(I_{0}\right)} \cdot p_{2}\left(I_{0}\right) d I_{0}
$$

\footnotetext{
${ }^{2}$ The current is time averaged over a period of $N \cdot T$.
}

If we substitute $\mu$ using Eqn. (2.5), Eqn. (3.1) becomes:

$$
P^{a c t}=V_{0} \cdot \int_{I_{0, M I N}}^{I_{0, M A X}} \frac{I_{0}}{1-\beta \cdot I_{0}} \cdot p_{2}\left(I_{0}\right) d I_{0}
$$

Under the constraint of a fixed mean value $I_{0}^{\text {ave }}$, it is easy to prove that the maximum $P^{a c t}$ occurs when $I_{0}$ follows a uniform distribution, i.e.,

$$
p_{2}\left(I_{0}\right)=\left\{\begin{array}{cl}
\frac{1}{I_{0, M A X}-I_{0, M I N}}, & I_{0, M I N} \leq I_{0} \leq I_{0, M A X} \\
0, & \text { otherwise }
\end{array}\right.
$$

The minimum $P^{a c t}$ occurs when $I_{0}$ follows a Dirac's $\delta$-function distribution, i.e.,

$$
p_{2}\left(I_{0}\right)=\delta\left(I_{0}-I_{0}^{\text {ave }}\right)
$$

Substituting (3.3) and (3.4) into (3.2) respectively, we obtain:

$$
\begin{aligned}
P_{M A X}^{a c t} & =V_{0} \cdot \int_{I_{0, M I N}}^{I_{0, M A X}} \frac{I_{0}}{1-\beta \cdot I_{0}} \cdot \frac{1}{\left(I_{0, M A X}-I_{0, M I N}\right)} d I_{0} \\
& =\frac{V_{0}}{\left(I_{0, M A X}-I_{0, M I N)}\right.} \cdot \frac{\beta\left(I_{0, M I N}-I_{0, M A X}\right)+\ln \left(\frac{1-\beta \cdot I_{0, M I N}}{1-\beta \cdot I_{0, M A X}}\right)}{\beta^{2}}
\end{aligned}
$$

and

$$
\begin{aligned}
& P_{M I N}^{a c t}=V_{0} \cdot \int_{I_{0, M I N}}^{I_{0, M A X}} \frac{I_{0}}{1-\beta \cdot I_{0}} \cdot \delta\left(I_{0}-I_{0}^{\text {ave }}\right) d I_{0} \\
& =\frac{V_{0} \cdot I_{0}^{\text {ave }}}{1-\beta \cdot I_{0}^{\text {ave }}}
\end{aligned}
$$

If we use Eqn. (2.6) instead, Eqn. (3.1) can be written as:

$$
P^{a c t}=V_{0} \cdot \int_{I_{0, M I N}}^{I_{0, M A X}} \frac{I_{0}}{1-\gamma \cdot I_{0}^{2}} \cdot p_{2}\left(I_{0}\right) d I_{0}
$$

and we obtain the following expressions for the maximum and minimum $P^{a c t}$ :

$$
P_{M A X}^{a c t}=\frac{V_{0}}{\left(I_{0, M A X}-I_{0, M I N}\right)} \cdot \frac{\ln \left(\frac{1-\gamma \cdot I_{0, M I N}^{2}}{1-\gamma \cdot I_{0, M A X}^{2}}\right)}{2 \gamma}
$$

and

$$
P_{M I N}^{a c t}=\frac{V_{0} \cdot I_{0}^{\text {ave }}}{1-\gamma \cdot\left(I_{0}^{\text {ave }}\right)^{2}}
$$

\section{B. An example}

To provide a quantitative example, we assign $V_{0}=4 \mathrm{~V}, I_{0, M I N}=0$, $I_{0, M A X}=5 \mathrm{~A}, \beta=0.12, \gamma=0.024$, and $C A P_{0}=36 \mathrm{KJ}$ (2.5 Amp-Hour at $4.0 \mathrm{~V}$ output voltage). Notice that the values of $\beta$ and $\gamma$ are chosen such that both (2.5) and (2.6) evaluate to 0 when $I_{o}=0$ and evaluate to 0.4 when $I_{o}=5 \mathrm{~A}$. Figure 5 and Figure 6 give several simple distributions with the same mean value of $2.5 \mathrm{~A}$ for the discharge current $^{3}$. The current profiles in Figure 5 are representative of the current profile for a circuit which is operating in one stable mode (uni-modal operation). The current profiles in Figure 6 are

\footnotetext{
${ }^{3}$ In the drawings, the magnitudes of the pulses/peaks are not shown to scale.
} 
representative of the current profile for a circuit which is operating alternatively between two stable modes (bi-modal operation). The phenomenon of bi-modal operation may be caused by input characteristics of the circuit, the scheduling of the tasks, or dynamic power management. Table 1 and Table 2 give the corresponding duration-of-service when using Eqn.(2.5) and Eqn. (2.6) for $\mu$.

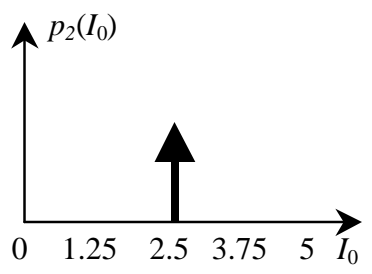

(1) Pulse

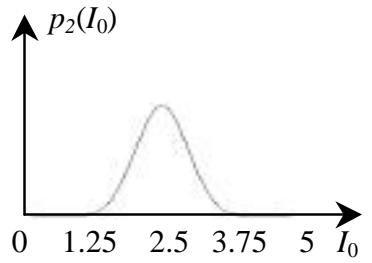

(3) Normal $(\sigma=0.5)$

Figure 5 Current profiles for uni-modal operation.
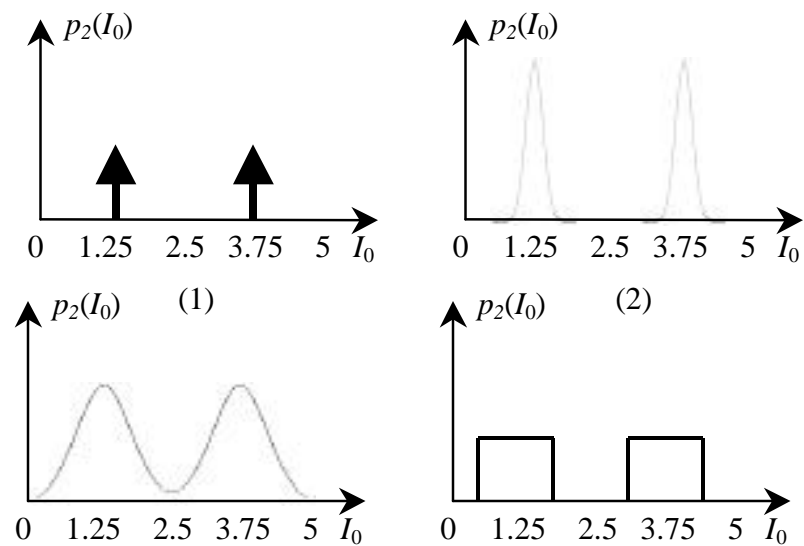

(3)

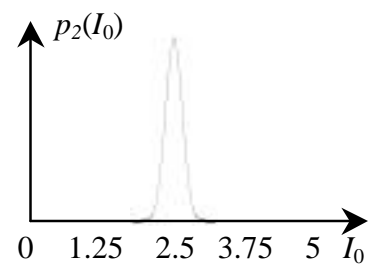

(2) Normal $(\sigma=0.1)$

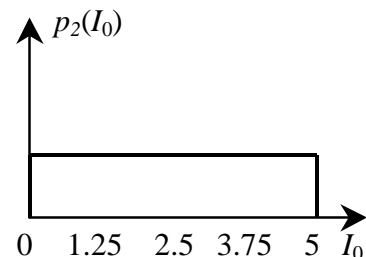

(4) Uniform

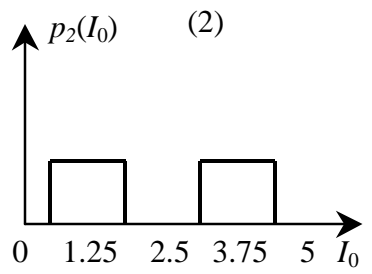

(4)
Figure 6 Current profiles for bi-modal operation.

Table 1 Battery lifetime for uni-modal current profile.

\begin{tabular}{|c|l|c|c|c|c|}
\hline Profile & & 1 & 2 & 3 & 4 \\
\hline \multirow{2}{*}{$D O S$ (hour) } & Eqn. (2.5) with $\beta=0.12$ & 0.70 & 0.70 & 0.68 & 0.57 \\
\cline { 2 - 7 } & Eqn. (2.6) with $\gamma=0.024$ & 0.85 & 0.85 & 0.83 & 0.65 \\
\hline
\end{tabular}

Table 2 Battery lifetime for bi-modal current profile.

\begin{tabular}{|c|l|c|c|c|c|}
\hline Profile & & 1 & 2 & 3 & 4 \\
\hline \multirow{2}{*}{$D O S$ (hour) } & Eqn. (2.5) with $\beta=0.12$ & 0.60 & 0.60 & 0.59 & 0.59 \\
\cline { 2 - 6 } & Eqn. (2.6) with $\gamma=0.024$ & 0.72 & 0.72 & 0.70 & 0.70 \\
\hline
\end{tabular}

From the results in Table 1 and Table 2, we conclude that,

1. The maximum DOS occurs by using the $\delta$-function distribution whereas the minimum DOS occurs by using the uniform distribution. There is a significant increase $(20 \%$ $30 \%$ ) in DOS from the worst case to the best case.
2. With $\delta$-function current distribution, a circuit with bi-modal current distribution exhausts the battery more rapidly compared to one with the same average current but a unimodal operation. The opposite is true for uniform uni-modal versus bi-modal current distributions.

3. The variation of DOS from best case to worst case current profile is much higher for the uni-modal operation compared with the bi-modal operation.

\section{MINIMIZING THE PRODUCT OF BATTERY-DISCHARGE AND DELAY}

In the past, the energy-delay metric was used to find an optimal supply voltage $V_{d d}$ for the best power-performance tradeoff. Here we propose another metric for low power design in an integrated battery-hardware model, the battery discharge-delay product. This metric is similar to the energy-delay product while accounting for the battery characteristics and the DC/DC conversion efficiency. The $B D$-delay product states that the design goal should be to minimize delay and maximize battery lifetime at the same time.

The problem of static voltage scaling for a battery-power system is defined as: Given a battery with certain characteristics, a DC/DC converter with certain efficiency, and a design of CMOS circuit, find the optimal supply voltage $V_{d d}$ for the CMOS circuit such that the $B D$-delay product is minimized.

\section{A. The BD-delay product}

We define the Battery Discharge $(B D)$ as:

$$
B D=\frac{E^{a c t}}{C A P_{0}}
$$

As we discussed in previous section, $E^{a c t}$ will be different for different current profiles. For convenience of presentation, we assume that the current distribution follows the simplest (and best) profile i.e., a $\delta$-function distribution as shown in Fig. 5.(1) Obviously, other current distributions could be used instead. Therefore, we have:

$$
B D=\frac{E^{a c t}}{C A P_{0}}=\frac{V_{0} \cdot I_{0} \cdot T}{C A P_{0} \cdot \mu\left(I_{0}\right)}
$$

The ideal energy needed for circuit to complete an operation is [2]:

$$
E^{i d e}=V_{d d} \cdot I_{d d} \cdot T=\frac{1}{2} C_{s w} \cdot V_{d d}^{2}
$$

where $C_{s w}$ is the total switched capacitance during the operation.

From Equations (2.1), (4.2) and (4.3), we can write BD as a function of $V_{d d}$ :

$$
B D=\frac{C_{s w}}{2 \cdot \eta \cdot C A P_{0}} \cdot \frac{V_{d d}^{2}}{\mu\left(k \cdot V_{d d}^{2} / T\right)}
$$

where $k=C_{s w} /\left(2 \cdot \mu \cdot V_{0}\right)$.

Either (2.5) or (2.6) can substitute the efficiency function $\mu$ in (4.4). Without loss of generality, we only use (2.5) for the rest of our discussion.

Substituting (2.5) in (4.4), we obtain:

$$
B D=\frac{C_{s w}}{2 \cdot \eta \cdot C A P_{0}} \cdot \frac{V_{d d}^{2}}{\left(1-\beta \cdot k \cdot V_{d d}^{2} / T\right)}
$$


For today's deep sub-micron CMOS technology, the delay of a circuit can be modeled as:

$$
t_{d}=m \frac{V_{d d}}{\left(V_{d d}-V_{t h}\right)^{\alpha}}, \quad 1<\cdot \leq 2
$$

where $m$ is some constant and $V_{t h}$ is the threshold voltage of the transistor. Notice that Eqn. (4.6) can be used for modeling the delay of the whole circuit, as well as a single gate.

We can thus write the $B D$-delay $(B D-D)$ product as:

$$
B D-D=\frac{m \cdot C_{s w}}{2 \cdot \eta \cdot C A P_{0}} \cdot \frac{V_{d d}^{3}}{\left(1-\beta \cdot k \cdot V_{d d}^{2} / T\right) \cdot\left(V_{d d}-V_{t h}\right)^{\alpha}}
$$

When we are calculating the optimal $V_{d d}$ that minimizes the $B D-D$ product, we need to consider two different cases on $T$ :

1. Fixed operation latency: $T$ is constant for all $V_{d d}$ values. In this case, Eqn. (4.7) can be used to calculate the optimal $V_{d d}$.

2. Variable operation latency: $T$ changes when $V_{d d}$ changes. In this case, it is reasonable to assume that $T$ is proportional to operation delay:

$$
T \propto t_{d} \Rightarrow T=m^{\prime} \frac{V_{d d}}{\left(V_{d d}-V_{t h}\right)^{\alpha}}, \quad 1<\cdot \leq 2
$$

Therefore, the $B D-D$ product is written as:

$$
B D-D=\frac{m \cdot C_{s w}}{2 \cdot \eta \cdot C A P_{0}} \cdot \frac{V_{d d}^{3}}{\left(1-\beta \cdot k \cdot V_{d d} \cdot\left(V_{d d}-V_{t h}\right)^{\alpha} / m^{\prime}\right) \cdot\left(V_{d d}-V_{t h}\right)^{\alpha}}
$$

We will see in the next section, although the optimal $V_{d d}$ values calculated by (4.7) and (4.8) are different, they have similar characteristics.

\section{B. Quantitative examples}

\section{Fixed Operation Latency (FOL)}

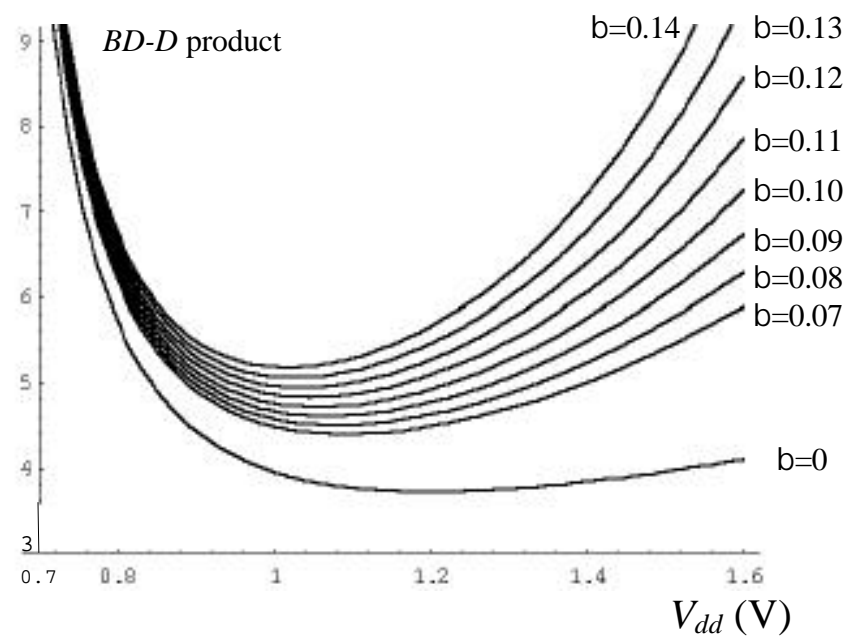

Figure $7 B D-D$ product curves with different $\beta$ values (FOL).

Assume a VLSI circuit consumes $13.5 \mathrm{~W}$ power at supply voltage of $V_{d d}=1.5 \mathrm{~V}$. Let $V_{0}=4 \mathrm{~V}$ and $\eta=0.9$. We have $k / T=1.7$. Let $\alpha=1.5$, and $V_{t h}=0.6 \mathrm{~V}$. We normalized $\left(m \cdot C_{s w}\right) /\left(2 \cdot \eta \cdot C A P_{0}\right)=1$ since their values will not influence the optimal $V_{d d}$ and the shape of $B D-D$ product. To show the influence of the battery characteristics on the optimal $V_{d d}$, we use $\beta$ values of $(0,0.07,0.08,0.09,0.1,0.11$,
$0.12,0.13,0.14)$ to generate a group of $B D-D$ product curves and compare the optimal $V_{d d}$ values. Notice that if $\beta=0$, the $B D-D$ product is equivalent to the ideal case where the energy-delay product is calculated without considering the battery characteristics. Figure 7 shows the plot of $B D-D$ product curves with different $\beta$ values. Table 3 shows the corresponding optimal $V_{d d}$ values.

Table 3 Optimal $V_{d d}$ for minimum BD-D product (FOL).

\begin{tabular}{|c|c|c|c|c|c|c|c|c|}
\hline$\beta$ & 0 & 0.08 & 0.09 & 0.10 & 0.11 & 0.12 & 0.13 & 0.14 \\
\hline Optimal $V_{d d}(\mathrm{~V})$ & 1.200 & 1.080 & 1.068 & 1.057 & 1.047 & 1.037 & 1.027 & 1.018 \\
\hline
\end{tabular}

\section{Variable Operation Latency (VOL)}

The parameter settings are same as in the case of fixed operation latency, except that $\mathrm{k} / \mathrm{m}^{\prime}$ (instead of $\mathrm{k} / \mathrm{T}$ ) is calculated to be 3.0. Figure 8 shows the plot of $B D-D$ product curves with different $\beta$ values. Table 4 shows the corresponding optimal $V_{d d}$ values.

Table 4 Optimal $V_{d d}$ for minimum $B D-D$ product (VOL).

\begin{tabular}{|c|c|c|c|c|c|c|c|c|}
\hline$\beta$ & 0 & 0.08 & 0.09 & 0.10 & 0.11 & 0.12 & 0.13 & 0.14 \\
\hline Optimal $V_{d d}(\mathrm{~V})$ & 1.200 & 1.073 & 1.063 & 1.054 & 1.046 & 1.038 & 1.031 & 1.024 \\
\hline
\end{tabular}

The results in Tables 3 and 4 show that the optimal $V_{d d}$ for minimum $B D-D$ product in an integrated battery-hardware model can differ by about $10 \%$ to $15 \%$ from the one which does not consider the battery characteristics. The optimal $V_{d d}$ will decrease when $\beta$ increases.

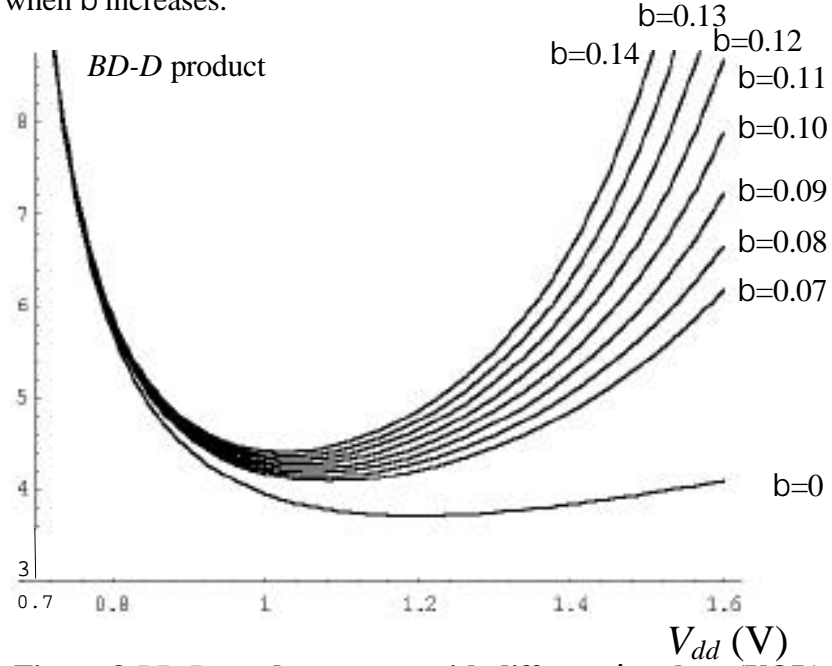

Figure $8 B D-D$ product curves with different $\beta$ values (VOL).

\section{EXPERIMENTAL RESULTS}

Experiments using HSPICE simulation are designed to verify our analysis in previous sections.

A macro-model of the battery was generated following the model proposed by [16]. The parameters in the macro-model were set according to the data sheet of a commercial lithium battery [17]. The capacity of the battery is 3 Amp-Hour and the output voltage is $3.8 \mathrm{~V}$. The capacity-current characteristic of the battery has been shown in Figure 4. N.T is set to be 6 seconds.

An appropriate macro-model was used for the DC/DC converter simulation. The efficiency of the converter was set to $90 \%$ for converting $V_{0}$ to different $V_{d d}$ 's. 
Seven different profiles for the battery discharge current are generated. They are:

1) $\delta$-function distribution with mean of $1.5 \mathrm{~A}$

2) Normal distribution with mean of $1.5 \mathrm{~A}$ and $\sigma=0.1$

3) Normal distribution with mean of $1.5 \mathrm{~A}$ and $\sigma=0.5$

4) Uniform distribution over region $[0,3]$

5) Bi-modal $\delta$-function distributions with means of $0.25 \mathrm{~A}$ and 2.75A for each mode

6) Bi-modal $\delta$-function distributions with means of $0.5 \mathrm{~A}$ and $2.5 \mathrm{~A}$ for each mode

7) Bi-modal $\delta$-function distributions with means of $1 \mathrm{~A}$ and $2 \mathrm{~A}$ for each mode

The simulated duration of service (or battery lifetime) for different current profiles are reported in Table 5. The experimental results are consistent with our analysis.

Table 5 Simulation results of $D O S$ for different profiles

\begin{tabular}{|c|c|c|c|c|c|c|c|}
\hline Profile & 1 & 2 & 3 & 4 & 5 & 6 & 7 \\
\hline DOS (hours) & 1.77 & 1.72 & 1.49 & 1.42 & 1.46 & 1.52 & 1.64 \\
\hline
\end{tabular}

For the experimental setup of the $B D$-delay product (variable operation latency), we designed a small system where the VLSI circuit is represented by an optimally sized 4-inverter buffer with a capacitive load of $0.5 \mathrm{pF}$. A $0.35 \mu$ CMOS process technology (BSIM3 models) [18] is used for the transistor models. Several supply voltages ranging from $0.8 \mathrm{~V}$ to $1.6 \mathrm{~V}$ are used for the buffer. For each supply voltage, delay and average current are measured for the buffer to make a single transition. The delay values are directly used in the final $B D$-delay product. We scale-up the average current by a factor of 15,000 to create a more realistic discharge current profile representative of a VLSI circuit. Then we use the average current as the battery discharge current to get the values of $B D$. The simulated $B D-D$ product curve is shown in Figure 9. The simulated optimal $V_{d d}$ value for minimum $B D-D$ product is $0.9 \mathrm{~V}$ for the battery model we use.

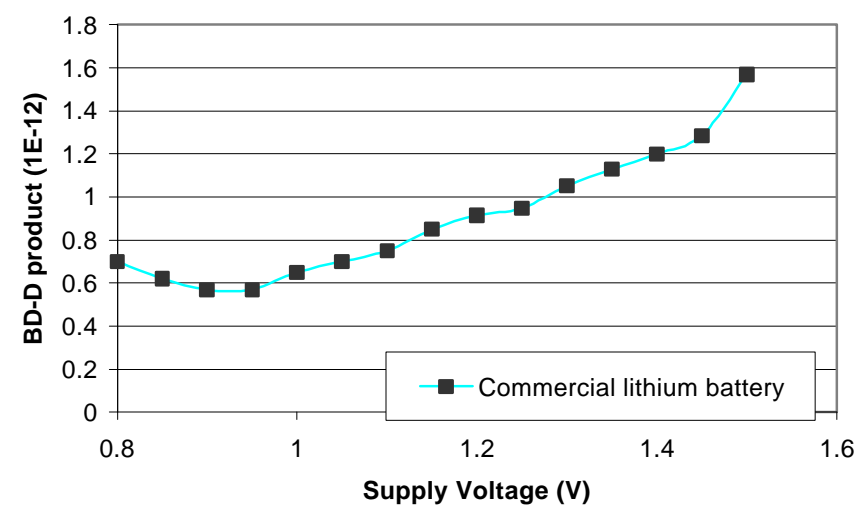

Figure 9 Experimental results of the $B D-D$ product curve.

By our analysis and experiments, some implications for low power design of battery-powered devices are:

1. Current profile has a significant impact on the duration of service of the battery. When we are designing or optimizing a circuit for low power, we must consider both the average current dissipation and the variance of the average current

2. The incorporation of real battery characteristics in the low power design analysis necessitates the use of even lower supply voltages by pushing the optimal $V_{d d}$ (for minimum $B D-D$ product) lower than was initially thought [2]. Using an integrated battery-hardware model, we can see that, achieving higher circuit performance by increasing the supply voltage level is even costlier than previously thought.

\section{CONCLUSIONS}

In this paper, we showed that it is essential to consider the characteristics of the battery that powers a portable electronic circuit in deciding the effectiveness of various low power optimization techniques. We also proposed a simple, yet accurate, integrated model of the battery and VLSI sub-systems. Then we studied the relationship between battery lifetime and different current distributions. Next we studied the problem of assigning a voltage level to the VLSI circuit which minimizes the product of delay and the battery discharge in the combined system. Finally we give some suggestions for low power design for batterypowered devices.

\section{REFERENCES}

[1] A. Chandrakasan, R. Brodersen, Low Power Digital CMOS Design, Kluwer Academic Publishers, July 1995.

[2] M. Horowitz, T. Indermaur, and R. Gonzalez, "Low-Power Digital Design”, IEEE Symposium on Low Power Electronics, pp.8-11, 1994.

[3] A. Chandrakasan, V. Gutnik, and T. Xanthopoulos, "Data Driven Signal Processing: An Approach for Energy Efficient Computing", 1996 International Symposium on Low Power Electronics and Design”, pp. 347-352, Aug. 1996.

[4] J. Rabaey and M. Pedram, Low Power Design Methodologies, Kluwer Academic Publishers, 1996

[5] URL: http://infopad.eecs.berkeley.edu/ anthonys/quals

[6] M. Pedram and Q. Wu, "Battery-Powreed Digital CMOS Design", Proceedings of Design Automation and Test in Europe Conference, pp. 72-76, Mar., 1999.

[7] M. Pedram, "Power Minimization in IC Design: Principles and Applications", ACM transactions on Design Automation of Electronic Systems, Vol. 1, No. 1, pp. 3-56, Jan., 1996.

[8] M. Doyle, T. F. Fuller, and J. Newman, "Modeling of Galvanostatic Charge and Discharge of the Lithium/Polymer/Insertion Cell", $J$. Electrochem. Soc., Vol. 140, No. 6, pp.1526-1533, Jun. 1993.

[9] T. F. Fuller, M. Doyle, and J. Newman, "Simulation and Optimization of the Dual Lithium Ion Insertion Cell", $J$. Electrochem. Soc., Vol. 141, No. 1, pp.1-9, Jan. 1994.

[10] D. Fauteux, "Lithium Polymer Electrolyte Rechargeable Battery", The Electrochemical Society Proceedings, Vol. 94-28, pp.379-388.

[11] L. Xie, W. Ebner, D. Fouchard, and S. Megahed, "Electrochemical Studies of $\mathrm{LiNiO}_{2}$ for Lithium-Ion Batteries", The Electrochemical Society Proceedings, Vol. 94-28, pp.263-276.

[12] K. M. Abraham, D. M. Pasquariello, T. H. Nguyen, Z. Jiang, and D. Peramunage, "Lithiated Manganese Oxide Cathodes for Rechargeable Lithium Batteries", The Battery Conference, pp. 317323, 1996.

[13] N. Cui, B. Luan, D. Bradhurst, H. K. Liu, and S. X. Dou, "SurfaceModified $\mathrm{Mg}_{2} \mathrm{Ni}$-Type Negative Electrode Materials for $\mathrm{Ni}-\mathrm{MH}$ Battery", The Battery Conference, pp. 317-322, 1997.

[14] J. K. Erbacher and S. P. Vukson, "Commercial Nickel-Metal Hydride (Ni-MH) Technology Evaluation”, The Battery Conference, pp. 9-15, 1997

[15] B. Nelson, "TMP Ultra-High Rate Discharge Performance", The Battery Conference, pp. 139-143, 1997.

[16] S. Gold, "A PSPICE Macromodel for Lithium-Ion Batteries", The Battery Conference, pp. 215-222, 1997

[17] URL: http://www.valence-tech.com/products/index.htm

[18] URL: http://www.mosis.org/html/hp-gmos10qa-prm.html 